\title{
Geo-Quantities: A Framework for Automatic Extraction of Measurements and Spatial Context from Scientific Documents
}

\author{
Thorge Petersen \\ Christian-Albrechts-University Kiel \\ Kiel, Germany \\ petersen@rz.uni-kiel.de \\ Hardik Patel \\ Dept. of Computational and Data \\ Sciences \\ George Mason University \\ Fairfax, Virginia, USA \\ hpatel12@gmu.edu
}

\author{
Muhammad Asif Suryani \\ Christian-Albrechts-University Kiel, \\ GEOMAR Helmholtz Centre for \\ Ocean Research Kiel \\ Kiel, Germany \\ mas@informatik.uni-kiel.de \\ Klaus Wallmann \\ GEOMAR Helmholtz Centre for \\ Ocean Research Kiel \\ Kiel, Germany \\ kwallmann@geomar.de
}

\author{
Christian Beth \\ Christian-Albrechts-University Kiel \\ Kiel, Germany \\ cbe@informatik.uni-kiel.de
}

\author{
Matthias Renz \\ Christian-Albrechts-University Kiel \\ Kiel, Germany \\ mr@informatik.uni-kiel.de
}

\begin{abstract}
Quantitative information derived from scientific documents provides an important source of data for studies in almost all domains, however, manual extraction of this information is very time consuming. In this paper we will introduce a system Geo-Quantities that supports the automatic extraction of quantitative, spatial and temporal information of a given measurement entity from scientific literature using text mining techniques. The difficulty of automatic measurement recognition is mainly caused by the diverse expressions in the papers. Geo-Quantities offers an interactive interface for the visualization of extracted user-defined information, in particular spatial and temporal context. In our demonstration, we will showcase the capabilities of our system by retrieving measurements such as "mass accumulation rates" and "sedimentation rates" from scientific publications in the field of marine geology, which could have high impact in studies for building global mass accumulation rate maps. For training and evaluation of Geo-Quantities we use a corpus of domain-relevant papers.
\end{abstract}

\section{CCS CONCEPTS}

- Applied computing $\rightarrow$ Document management and text processing; • Computing methodologies $\rightarrow$ Information extraction.

\section{KEYWORDS}

entity recognition, spatial and temporal data, text mining, geoquantities, mass accumulation rate

\section{ACM Reference Format:}

Thorge Petersen, Muhammad Asif Suryani, Christian Beth, Hardik Patel, Klaus Wallmann, and Matthias Renz. 2021. Geo-Quantities: A Framework

Permission to make digital or hard copies of part or all of this work for personal or classroom use is granted without fee provided that copies are not made or distributed for profit or commercial advantage and that copies bear this notice and the full citation on the first page. Copyrights for third-party components of this work must be honored

For all other uses, contact the owner/author(s).

SSTD '21, August 23-25, 2021, virtual, USA

(c) 2021 Copyright held by the owner/author(s).

ACM ISBN 978-1-4503-8425-4/21/08.

https://doi.org/10.1145/3469830.3470911 for Automatic Extraction of Measurements and Spatial Context from Scientific Documents. In 17th International Symposium on Spatial and Temporal Databases(SSTD '21), August 23-25, 2021, virtual, USA. ACM, New York, NY, USA, 4 pages. https://doi.org/10.1145/3469830.3470911

\section{INTRODUCTION}

The availability of data is rapidly increasing, while data driven research approaches still suffer from the problem that observations are often not collected in central accessible databases. For example in the Marine Science field the extraction of mass accumulation rates (MARs) at the seafloor are a good example. This fundamental parameter has been measured since more than one hundred years at numerous locations around the globe. However, the MAR data were never compiled in a database but reported in a very large number of individual publications.

In general, scientific publications are the primary source of information dissemination among research communities. With the increase in the number of scientific publications in every domain it is becoming more and more challenging for researchers to study and extract all relevant information, especially the quantitative information from inline text. Though, publications are well-structured and the inline text carries information in various forms, to study and pursue relevant information from these publications often requires plenty of efforts, time as well as domain expertise [2]. Quantitative information in scientific publications, like MAR, could be anywhere, and may appear in different arrangements as well as different sections of the source documents, e.g. inline text, tables, diagrams, metadata, etc. In the case of detecting measurements in scientific documents, the inline-text usually contains the textual elaborations of the findings as well as relevant quantitative information. An automatic extraction of such quantitative information could prove to become indispensable as it will quickly lead to the relevant information by reducing the processing time. Moreover, it also enhances and accelerates empirical activities [9].

Publications are usually well-written for human readers, but not for digital extractors. In order to identify and extract relevant quantitative information automatically there are a lot of factors 
that need to be considered, e.g. writing styles, variation of expressions and abbreviations [8] as well as the problem of matching the measurement entity, e.g. MAR, with the corresponding quantity information, like " $44 \mathrm{~g} \mathrm{~cm}^{-2} \mathrm{yr}^{-1}$ ". There are numerous ways to represent such information, which need to be considered accordingly and may broaden the scope of the process [1]. In addition, spatial and temporal tagging of quantities is very important in various fields of science as well, as they help in understanding the quantities and their characteristics in depth. In marine geology, for example, there are measurements that could also exhibit spatial characteristics beside quantitative information, e.g. mass accumulation rate and sedimentation rate. The reason we target these particular quantities is their importance in the respective field, as the extraction of these quantities may contribute towards future findings, which makes them a suitable candidate for experimentation [1] [12].

In this paper, we introduce a framework named Geo-Quantities, which focuses on the extraction of quantitative and spatial information from scientific publications. Furthermore, the extracted information will be illustrated by a visualisation module, which offers an interactive interface. Without loss of generality, the focus of this work will be on marine geology.

\section{RELATED WORK}

Parsing measurements in data sets across different domains is a well known research problem and many techniques have been proposed over the years to address it. Popular approaches include regular expressions, knowledge bases, supervised machine learning, and hybrid approaches. Regular expressions are usually combined with additional approaches, for example with knowledge bases such as thesauri or ontologies, however in such approaches the system must first be filled with available knowledge [13]. Recently in 2019, an unsupervised rule-based approach was proposed that identifies units in source data and provides a corresponding semantic representation based on NASA's QUDT (Quantity, Unit, Dimension and Type) ontology using Arpeggio as a grammar parser [11].

In a supervised machine learning-based approach, measurement parsing is usually formally defined as a sequence labelling problem encompassing a variety of tasks, e.g. part-of-speech (POS) tagging or named-entity recognition (NER) [13]. Most of the existing tools are trainable, which means that they are able to automatically learn complex features and adapt parsing rules from training data. On average, ML-based systems achieve better results in comparison to rule-based systems, especially in terms of recall, with conditional random fields (CRFs) being the most popular metadata extraction algorithm. A study on the parsing of bibliographic references showed that the most powerful standard tools are GROBID, followed by CERMINE and ParsCit. All of these tools implement CRF-based learning algorithms [13].

GROBID is an open source machine learning library for extracting, parsing and re-structuring raw documents into structured $\mathrm{XML} / \mathrm{TEI}$ encoded documents based on CRFs with the possibility to use various deep learning architectures for sequence labeling, such as BERT-CRF [10]. Furthermore, there exists already a GROBIDbased module called Grobid-quantities, which extracts physical measurement expressions from text documents and normalizes them to the base SI unit [8]. Similarly, GPT-3 [3] provides a model that is adaptive to specific scientific documents.

Marve is a framework built on Grobid-quantities, that extends existing techniques in NLP and word processing to extract the context around measurements in natural language, such as related entities and descriptors [9]. Marve uses Grobid-quantities to determine measurements, units and the "quantified" substance. CoreNLP is then used to link measurements to related words in the sentence using CoreNLP word dependencies and POS tags. Recently, Grobid-quantities has been used as an extraction module in various fields of science, e.g. the detection of astronomical entities [6], and the detection of superconducting material names and their critical temperature [7].

All the above stated applications are domain specific and only serve our purpose to some extent. Out-of-the-box it is not possible to detect location- or time-related context. So the idea here to test such systems for complex measurements i.e. mass accumulation rate and sedimentation rate which might contain derived measurement information as well as spatial and temporal context. So far, to the best of our knowledge there is no framework that can detect and extract measurements with associated spatial and temporal references from text.

\section{PROPOSED SYSTEM}

Our proposed framework Geo-Quantities, as shown in Figure 1, focuses on the main tasks, i.e. the extraction and visualization phase. The extraction module focuses on the intermediate steps regarding extraction of quantitative, spatial and temporal information (if available) from text sources. The visualization module is responsible for combining all the information from the extraction module and visualize it in an interactive and informative way to users.

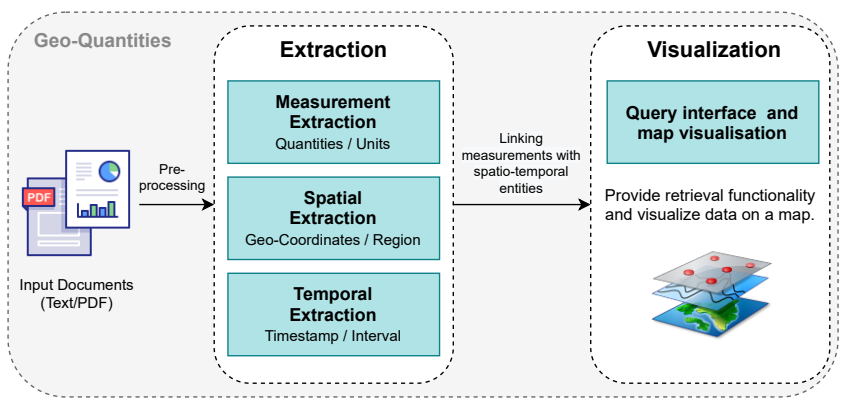

Figure 1: Geo-Quantities system overview.

Since we deal with scientific publications, with PDF being the primary publication format, the ability to process PDF documents is essential. Information can be extracted from the headings, in the running text, or in tables and figures. The input data is therefore pre-processed, and the titles, sections and paragraphs are parsed, whereby we first focus on the inline text. Apart from software that only offers certain analysis functions, there are also tools with which metadata and logical structures can be generically extracted from PDF, e.g. the GROBID framework on which our implementation is based. It has full support for extracting data from PDF and a well-documented integrated workflow for preregistering training 
data, as well as training and evaluating ML models. Among the various tools available, the choice of GROBID is particularly justified by the fact that the project is open source, under active development, and can be considered as production ready. Deployments in production include ResearchGate, HAL Research, CERN, and many others [10].

\subsection{Extraction}

The extraction module incorporates the pre-processed text as input, so to carry out the measurement extraction and spatial and temporal extraction in parallel. For context extraction, analogous to the conception of Grobid-quantities, Grobid-astro or similar extensions, we have created a new model only with labels regarding our domain-relevant location or temporal information and apply this cascaded to sections that are identified by GROBID's full-text model, so that the training data for your new labels are independent of the training data for the full-text model. The extraction of measurements, i.e. quantities and units, is based on the Grobidquantities module. The module's unit CRF model operates at the character level and uses a unit lexicon to highlight known units or prefixes [8]. Even more complex, unknown units can be extracted by deriving simple units.

While the CRF models of the module achieve good evaluation results with respect to many measurements - mostly with common units - the detection of various specific units, such as the MAR, is not directly possible. For example, the frequent use of the unit $y r$ (year) is not detected by the default model, so that from input texts such as $42 \mathrm{~g} \mathrm{~m}^{-2} \mathrm{yr}^{-1}$ only the first part is recognized, resulting in $42 \mathrm{~g} \mathrm{~m}^{-2}$. This is due to the variety of different expressions of MAR units in the scientific publications, missing annotation schemes and proper MAR unit handling as well as the lack of corresponding annotations in the Grobid-quantities default training data. The 35 scientific documents from various domains, which were used for training and evaluating the CRF models of Grobidquantities, obviously cannot contain sufficient annotation of all domain-specific complex units. In their analysis, the authors of the module themselves identify the need to provide more complex examples in the training data sets, because it turns out that the distribution is skewed towards simple units [8]. Statistically, these simple units occur more frequently and with less variability.

For this reason, we first expanded the module by adding new training data using a corpus of scientific publications relevant to the application area, and then annotated the expressions of the MAR and sedimentation rates in the generated TEI training set. We target occurring terms MARs and sedimentation rates with units that consist of combinations of units for mass $(\mathrm{mg}, \mathrm{g}, \mathrm{kg})$, area $\left(\mathrm{mm}^{2}, \mathrm{~cm}^{2}, \mathrm{~m}^{2}\right)$, time ( $\left.\mathrm{yr}, \mathrm{Kyr}, \mathrm{a}, \mathrm{Ka}, \mathrm{Ma}\right)$ and length $(\mathrm{mm}, \mathrm{cm}$, $m$ ). An example of a MAR measurement could be $42 \mathrm{~g} \mathrm{~cm}^{-2} \mathrm{yr}^{-1}$, a sedimentation rate could be $42 \mathrm{~cm} \mathrm{yr}^{-1}$. The necessary custom units for the annotation process and the normalization step are handled by the external Java library Unit of Measurement [4]. The newly trained models are then used for the measurement extraction.

For the extraction of spatial features from documents, the entityfishing module based on Grobid-ner handles the recognition of various entities related to places and/or well-defined regions, among others [5]. With locational features extracted from the document,

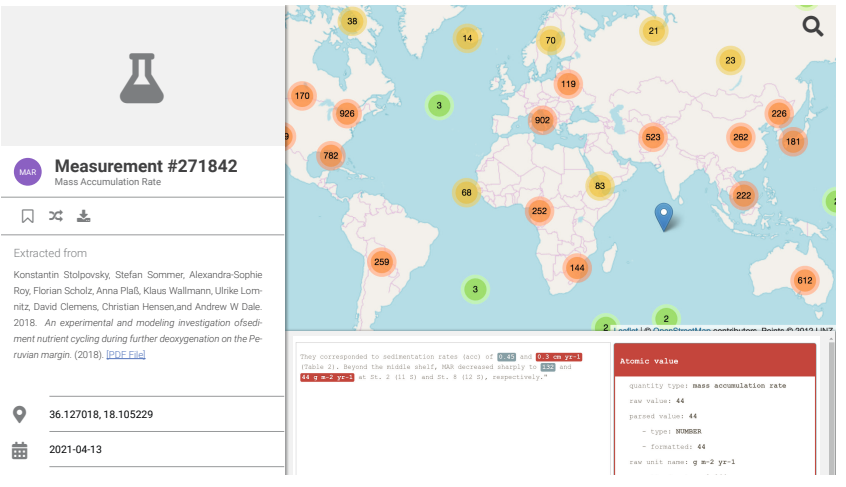

Figure 2: Prototype interface.

the user can thus explore spatial patterns and gain more insights from the actual data.

Finally, all classified measurements will be appropriately linked to the extracted location and time information, by adding the labels that appear closest to the measurements. If no close context information is identified, we use fallback data such as the year and place of publication.

\subsection{Visualization}

The visualization component (user interface) covers the primary use cases, namely a) spatial selection of measurements shown on the map $b$ ) showing the measurement details and metadata, and c) showing the referenced sections in the respective documents and highlighting the corresponding entities. Therefore, the contextenriched measurements are visualized on an interactive map, which enables direct access to the extracted information and the referenced source documents.

The measurements can also be filtered based on the linked time information. A prototype interface is illustrated in Figure 2.

\subsection{Architecture}

The architecture of our proposed system is shown in Figure 3 and follows a monolithic approach, although all components have clearly defined interfaces and could therefore be expanded for microservice-oriented operation.

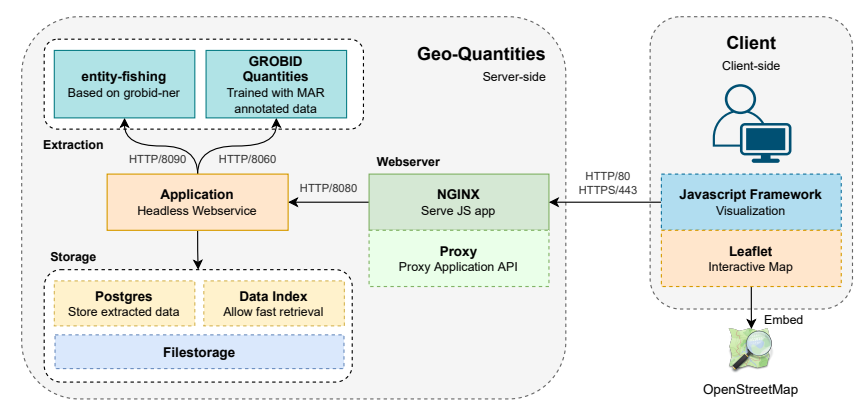

Figure 3: Proposed system architecture.

The extraction is carried out on the server side, with identified entities being linked accordingly by the main application and, depending on the data type, stored in a corresponding database and 
indexed for faster querying. This extraction module based on GROBID can be modified to be used with a pre-trained GPT- 3 model, as well, e.g. for a comparative study on entity extraction methods. The application itself is designed as a web service. We have largely designed a headless application that provides a corresponding API that allows for interaction with the web service. On the client side, a reactive application accesses the interface described. It provides the user interface with all measurements populated on an interactive map view based on the open source JavaScript library Leaflet.

\section{DEMONSTRATION}

The demonstration focuses on the effectiveness and scope of GeoQuantities. The primary objective of the proposed work is to develop a framework, which can extract complex measurements and their linked information from domain-specific text, i.e. relevant scientific publications.

In our scenario, we consider marine geology related measurements to test the significance of our proposed framework. Normally the picked measurements, i.e. mass accumulation rates and sedimentation rates, are collected at measurement stations or vessels at various geographical locations and assembled in scientific publications. Hence the extraction of such information from inline text is not the only case here, but we also need to handle and structure the necessary information linked with each extracted value. As a result, these values could have great impact and importance for later relevant experiments. We believe Geo-Quantities will prove useful for many researchers since this framework can provide them targeted measurements, e.g. MARs, and its context by linked information. Geo-Quantities can provide answers to questions including (but not limited to): Where has MAR been measured? What are the values of these readings? When were the MAR readings taken? What are related articles or references (metadata)? Moreover, the proposed visualization module will incorporate the measurements as well as any recognized associated spatial and temporal information and populate it on an interactive map, in order to concisely present useful information for domain experts. We envision to use Geo-Quantities to create a map of MARs. This MAR map would benefit the whole marine geology research community and could bring new features to light as well as it could help the research community to solve the more complex puzzles.

\section{SUMMARY AND FUTURE WORK}

The proposed framework tackles a challenging and exciting problem due to its diversity, since it targets heterogeneous measurements, which exhibit quantitative and spatial as well as temporal context. Furthermore, Geo-Quantities also showcases the extracted information in an interactive interface for a visual experience.

Geo-Quantities could emerge as an potential candidate in numerous fields of science in various capacities. Initially it can be used to handle domain specific measurements and in the future be extended by integrating general NER approaches, which could further enhance its reach and make it more adoptable. Furthermore, extraction module can be enhanced to do automatic extraction of measurements by automatic rule learning methods that exploit different features of scientific text.

\section{ACKNOWLEDGMENTS}

This work was supported by the Helmholtz School for Marine Data Science (MarDATA) partially funded by the Helmholtz Association (grant HIDSS-0005).

\section{REFERENCES}

[1] Gowtham Atluri, Anuj Karpatne, and Vipin Kumar. 2018. Spatio-temporal data mining: A survey of problems and methods. ACM Computing Surveys (CSUR) 51, 4 (2018), 1-41.

[2] Soumia Lilia Berrahou, Patrice Buche, Juliette Dibie-Barthelemy, and Mathieu Roche. 2013. How to extract unit of measure in scientific documents?. In KDIR: Knowledge Discovery and Information Retrieval. Springer, 454-459.

[3] Tom Brown, Benjamin Mann, Nick Ryder, Melanie Subbiah, Jared D Kaplan, Prafulla Dhariwal, Arvind Neelakantan, Pranav Shyam, Girish Sastry, Amanda Askell, Sandhini Agarwal, Ariel Herbert-Voss, Gretchen Krueger, Tom Henighan, Rewon Child, Aditya Ramesh, Daniel Ziegler, Jeffrey Wu, Clemens Winter, Chris Hesse, Mark Chen, Eric Sigler, Mateusz Litwin, Scott Gray, Benjamin Chess, Jack Clark, Christopher Berner, Sam McCandlish, Alec Radford, Ilya Sutskever, and Dario Amodei. 2020. Language Models are Few-Shot Learners. In Advances in Neural Information Processing Systems, H. Larochelle, M. Ranzato, R. Hadsell, M. F. Balcan, and H. Lin (Eds.), Vol. 33. Curran Associates, Inc., 1877-1901. https://proceedings. neurips.cc/paper/2020/file/1457c0d6bfcb4967418bfb8ac142f64a-Paper.pdf

[4] G. Contributors. 2015-2021. Units of Measurement. https://github.com/ unitsofmeasurement. Last access: July 13, 2021.

[5] G. Contributors. 2016-2021. entity-fishing. https://github.com/kermitt2/entityfishing.

[6] G. Contributors. 2016-2021. Grobid-Astro: A machine learning software for extracting astronomical entities from scholarly documents. https://github.com/ kermitt2/grobid-astro. Last access: July 13, 2021.

[7] Luca Foppiano, Thaer M Dieb, Akira Suzuki, and Masashi Ishii. 2019. Proposal for Automatic Extraction Framework of Superconductors Related Information from Scientific Literature. In 子情通信学会サビスコンピュティング研究 会 2019 年度第一回研究会, 第 43 回 MaDIS 研究交流会合同研究会. Tsukuba, Japan. https://hal.archives-ouvertes.fr/hal-02870896

[8] Luca Foppiano, Laurent Romary, Masashi Ishii, and Mikiko Tanifuji. 2019. Automatic Identification and Normalisation of Physical Measurements in Scientific Literature. In DocEng '19 - ACM Symposium on Document Engineering 2019. ACM Press, Berlin, Germany, 1-4. https://doi.org/10.1145/3342558.3345411

[9] Kyle Hundman and Chris Mattmann. 2017. Measurement Context Extraction from Text: Discovering Opportunities and Gaps in Earth Science. ArXiv abs/1710.04312 (10 2017).

[10] Patrice Lopez. 2009. GROBID: Combining Automatic Bibliographic Data Recognition and Term Extraction for Scholarship Publications. In Research and Advanced Technology for Digital Libraries, Maristella Agosti, José Borbinha, Sarantos Kapidakis, Christos Papatheodorou, and Giannis Tsakonas (Eds.), Vol. 5714. Springer Berlin Heidelberg, Berlin, Heidelberg, 473-474. https://doi.org/0.1007/978-3642-04346-8_62

[11] Basel Shbita, Arun Rajendran, Jay Pujara, and Craig A. Knoblock. 2019. Parsing, Representing and Transforming Units of Measure, In Proceedings of the Conference on Modeling the World's Systems (Pittsburgh, PA). Modeling the World's Systems.

[12] Konstantin Stolpovsky, Stefan Sommer, Alexandra-Sophie Roy, Florian Scholz, Anna Plaß, Klaus Wallmann, Ulrike Lomnitz, David Clemens, Christian Hensen, and Andrew W Dale. 2018. An experimental and modeling investigation of sediment nutrient cycling during further deoxygenation on the Peruvian margin. (2018).

[13] Dominika Tkaczyk, Andrew Collins, Paraic Sheridan, and Joeran Beel. 2018. Machine Learning vs. Rules and Out-of-the-Box vs. Retrained: An Evaluation of Open-Source Bibliographic Reference and Citation Parsers. In Proceedings of the 18th ACM/IEEE on joint conference on digital libraries. 99-108. https: //doi.org/10.1145/3197026.3197048 